\title{
Commentary Good night, sleep tight: the time is ripe for critical care providers to wake up and focus on sleep
}

\author{
Randall S Friese
}

Department of Surgery, University of Texas Southwestern Medical Center, 5323 Harry Hines Blvd Room E5.508, Dallas, Texas 75390 USA

Corresponding author: Randall S Friese, randall.friese@utsouthwestern.edu

Published: 12 May 2008

Critical Care 2008, 12:146 (doi:10.1186/cc6884)

This article is online at http://ccforum.com/content/12/3/146

(c) 2008 BioMed Central Ltd

See related research by Bourne et al., http://ccforum.com/content/12/2/R52

\begin{abstract}
The role of sleep during recovery from acute illness has been overlooked for decades. Advances in the support of critically ill patients have been made in mechanical ventilation, specialized nutrition support, highly specific antibiotic therapy, and early rehabilitation. However, the promotion of sleep - a basic tenet for survival - has been actively ignored by critical care providers. Bourne and coworkers recently conducted a small clinical trial that describes improved sleep efficiency with oral melatonin use in critically ill patients.
\end{abstract}

Most physicians, if not all, readily support the notion that achieving an adequate amount of quality sleep is essential for speedy recovery from acute illness, and promptly send their patients home with a prescription for adequate rest. However, when these acute illnesses require hospital admission, the importance of attaining adequate rest takes a back seat secondary to the necessities of running a hospital ward.

The practice of active sleep disruption is no more apparent than in the intensive care unit (ICU) setting, where lighting, noise, and frequent nocturnal assessments/treatments prevent acutely ill patients from attaining sleep of adequate quality. The time has come for critical care providers to give credence to the significance of 'prescribing' sleep in the inpatient setting, just as we prescribe antibiotics, nutrition support, deep venous ulcer prophylaxis, mechanical ventilation, gastric ulcer prophylaxis, and physical therapy.

In a previous issue of Critical Care, Bourne and coworkers [1] reported the results of a small randomized clinical trail in which they investigated the use of melatonin as a means to promote sleep in the critical care environment. Although the demonstrated effect of melatonin on sleep efficiency (as measured by bispectral index) was small, the study is extremely important. Most literature on sleep and recovery from illness provides either a description of the abnormal sleep patterns that occur in the ICU setting [2-4] or a review of the deleterious effects of sleep deprivation, with suggestions on ways to improve sleep in ICU patients [5-7]. Few authors have scientifically evaluated a potential way to promote sleep, as have Bourne and colleagues.

Research into the effects of active sleep promotion in the ICU is lacking. As we continue to improve the quality of critical care interventions, we must not overlook the importance of supporting the body's basic needs, namely nutrients, exercise, and sleep. The causes of sleep deprivation in the ICU are multifactorial. Therefore, research into sleep promotion should utilize a tool that is multifaceted, addressing sleep promotion at several different levels simultaneously. This tool must address noise and lighting issues, minimize nocturnal assessments and treatments, utilize pharmacologic interventions to enhance time in restorative sleep stages, and consider behavioral interventions that enhance sleep quality (for instance, relaxation techniques, massage, biofeedback, and music therapy).

Finally, if sleep promotion is to be effective in the ICU setting, then a paradigm shift must occur at all levels of the health care team. Physicians and ICU directors must develop and put into practice protocols for sleep promotion. Nursing staff and other bedside care providers must carefully follow these protocols. ICU monitoring practices must change.

When we fully recognize the importance of good quality sleep during recovery from acute illness and begin actively to promote sleep in the ICU setting, I believe that outcomes including infection rates, ICU and hospital lengths of stay, overall complication rates, and (most importantly) mortality rates - will improve. 


\section{Competing interests}

The author declares that they have no competing interests.

\section{References}

1. Bourne RS, Mills GH, Minelli C: Melatonin therapy to improve nocturnal sleep in critically ill patients: encouraging results from a small randomised controlled trial. Crit Care 2008, 12: R52.

2. Cooper AB, Thornley KS, Young GB, Slutsky AS, Stewart TE, Hanly PJ: Sleep in critically ill patients requiring mechanical ventilation. Chest 2000, 117:809-818.

3. Friese RS, Diaz-Arrastia R, McBride D, Frankel H, Gentilello LM: Quantity and quality of sleep in the surgical intensive care unit: are our patients sleeping? J Trauma 2007, 63:1210-1214.

4. Aurell J, Elmqvist D: Sleep in the surgical intensive care unit: continuous polygraphic recording of sleep in nine patients receiving postoperative care. $B M J$ 1985, 290:1029-1032.

5. Friese RS: Sleep and recovery from illness and injury: A review of theory, current practice, and future directions. Crit Care Med 2008, 36:697-705.

6. Weinhouse GL, Schwab RJ: Sleep in the critically ill patient. Sleep 2006, 29:707-716.

7. Parthasarathy S, Tobin MJ: Sleep in the intensive care unit. Intensive Care Med 2004, 30:197-206. 